ORfTIOA, Revinta Hispenomerieana de Fabooffe

Vol. XIX, No. 87 (dieiembre 1987): 8-42

\title{
¿QUÉ DETERMINA LAS CONDICIONES DE VERDAD?*
}

Christopher Peacooke Universidad de Londres

Hay dos dimensiones del contenido proposicional de una oración dada o de un estado mental dado. Por una parte, hay una dimensión que lleva información acerca de cuestiones tales como las condiciones que pueden conducir a un pensador a aceptar tal contenido y sus consecuencias en el pensamiento una vez aceptado. Por otra parte, está la condición de verdad del contenido. Una investigación de la primera dimensión podría tener como fin una descripción adecuada de las condiciones bajo las cuales se aceptan de hecho ciertos contenidos, o podría tener como meta un enunciado correcto de las condiciones normativas relacionadas con esos contenidos. Yo me ocuparé aquí únicamente de las condiciones normativas: de aquí que por "condición de aceptación" de un contenido dado entenderé un enunciado correcto de una condición normativa acerca de ese contenido. Entonces surge una pregunta acerca de la relación entre esas dos dimensiones del contenido: ¿cuál es, en general, la relación entre las condiciones de aceptación de un contenido y su condición de verdad?

La palabra "contenido" que aparece en esta pregunta

- Christopher Peacocke cedió gentilmente a Crílica los derechos para esta versión en español. 
se usa aquí para algo que se conforma esencialmente con los mismos principios que Frege pensó que gobiernan a los pensamientos. ${ }^{1}$ Los contenidos se individuan por consideraciones de significado cognoscitivo: son los objetos de actitudes proposicionales; tienen valores de verdad absolutos sin relativización a ninguna otra cosa; son entidades compuestas, estructuradas; y es posible que diferentes pensadores juzguen, argumenten o tengan otras actitudes proposicionales respecto del mismo contenido exactamente. Que pueda haber algo que se conforme con todas esas condiciones fregeanas es algo controvertido. Pero la afirmación de que puede haberlo no es esencial para lo que yo voy a argumentar. Usaré la estructura fregeana únicamente para facilitar la formulación. Aun si la estructura fregeana se invalida, podemos todavía formular la cuestión que quiero plantear acerca de las relaciones entre condiciones de aceptación y condiciones de verdad. En cualquier teoría plausible habrá algo que sea el objeto de las actitudes proposicionales y en cualquier teoría plausible lo que se cree debe, de alguna manera, determinar un valor de verdad. Por lo tanto, en el aparato de cualquier teoría plausible podemos preguntar: ¿cuál es la relación entre las condiciones de aceptación de lo que se cree y las condiciones para que lo que se cree determine el valor de verdad verdadero?

Aun cuando de ninguna manera la probaré, argumentaré en favor de la conjetura de que hay una concepción de las condiciones de aceptación de un contenido según la cual esas condiciones de aceptación determinan su condición de verdad. Más específicamente, argumentaré en apoyo de esta conjetura:

1 Vease por ejemplo G. Frege, Logical Invertigations, traducido por P. T. Geach (Blackwell, Oxford, 1977). 
Hay una clase de contenidos cuyas condiciones de verdad están directamente determinadas por algunas de sus condiciones de aceptación; las condiciones de verdad de los contenidos fuera de esta clase están determinadas en última instancia por sus relaciones con los contenidos dentro de esta clase inicial.

En la siguiente sección argumentaré en favor de la conjetura en el caso de ciertos contenidos observacionales; en seguida, argumentaré en favor de ella en conexión con contenidos universalmente cuantificados.

La primera parte de la conjetura, como está expuesta, sería instantáneamente avalada por alguien que sostiene que la verdad misma se ha de elucidar como alguna forma de cumplimiento ideal de condiciones evidenciales: de hecho tal teórico sostendrá que la primera parte cubre todos los casos. Pero esta no es la manera en la que yo argumentaré en favor de la conjetura. Por una parte, no estableceré las condiciones de aceptación de un contenido solamente en términos de las acciones de un ser con capacidades mayores que, o diferentes de, las nuestras: tal enfoque carece de fuerza en sí mismo para iluminar las relaciones èntre nuestra aceptación de un contenido y sus condiciones de verdad. Por otra parte, tampoco recortaré las condiciones de verdad de tal manera que se garantice, antes de la investigación de cualquier tipo particular de contenido, que la conjetura debe ser correcta. No presupondré una teoría anti-realista de la verdad. Lo que apoyaré es algo que en una teoría completa tiene que establecerse mediante un argumento detallado acerca de las condiciones de aceptación reales y la condición de verdad pre-teorética del contenido, en ausencia de razones independientes para pensar que ellas son defectuosas. Tal argumento tiene que darse para cada tipo de contenido en nuestro repertorio conceptual. Podemos, sin embargo, esperar que haya algunas formas generales de argumento que se 
puedan usar para varios tipos diferentes de contenido al establecer la conjetura.

Necesitamos evitar la trivialización de la conjetura. Ella sería trivializada si admitiéramos como condición de aceptación de "los hombres son mortales" la condición de que debe aceptarse solamente si los hombres realmente son mortales: ¿y quién va a contradecir la corrección de ese requerimiento normativo? Las condiciones de aceptación relevantes deben, entonces, restringirse más - a aquellas para las cuales tenemos una explicación inmediata de cómo un pensador puede manifestar el hecho de que está siguiendo sus normas y no otras normas. Siempre que ofrezca condiciones de aceptación para un contenido particular intentaré mostrar que esta restricción adicional se cumple. Si la conjetura es correcta, desde luego, la conformidad con las normas de una condición de aceptación que usa la condición de verdad estrictamente, puede manifestarse después de todo: pero en la concepción que trazaré, la explicación de la manifestación de tal conformidad procede vía las condiciones de aceptación para las cuales tenemos una explicación directa de la manifestación.

Las condiciones de aceptación que discutiré, se refieren comúnmente, en parte, al mundo externo a la cabeza del pensador; no están restringidas a estados psicológicos que no tienen implicaciones para el entorno del sujeto. Por lo tanto, esas condiciones de aceptación para un contenido no deben identificarse con lo que muchos que han usado la noción llamarían el papel conceptual de un estado mental con ese contenido. ${ }^{2}$ Según

2 Harry Field, "Logic, Meaning and Conceptual Role", Journal of Philosophy, lxxiv (1977), 379-409, p. 380; Brian Loar, “Conceptual Role and Truth Conditions", en Notre Dame Journal of Formal Logic, xxiii (1982), 272-283, p. 280; vease ademán Colin McGinn, "The Structure of Content" , on Andrew Woodfield (ed.), Thought and Object (Clarendon Prew, Oxford, 1982); Stephen Schiffer, "Intention-based Semantica" 
tal noción interna del papel conceptual, el papel conceptual de un estado se refiere únicamente a la relación de ese estado con la estimulación, la conducta y otros estados internos: se sigue inmediatamente que, en casi todos los casos, tales papeles conceptuales internos por sí mismos no determinarán las condiciones de verdad, las cuales generalmente se referirán al mundo externo. El proyecto de elucidar las condiciones de aceptación, del que me ocuparé, y el proyecto de elucidar el papel conceptual interno, son distintos uno del otro.

Los dos proyectos pueden, por supuesto, rodearse de afirmaciones que hagan que los proyectos entren en conflicto. Un teórico del papel conceptual interno que dice que únicamente en el nivel del papel conceptual interno puede caracterizarse la dimensión del contenido que captura las condiciones de aceptación, estaría diciendo ciertamente algo que rivaliza con la explicación que yo ofreceré. ${ }^{3}$ Lo mismo ocurriría en el caso de un teórico - del papel conceptual interno o más amplioque afirmara que en la medida en que para él tiene sentido la posibilidad, la determinación de las condiciones de verdad mediante las condiciones evidenciales $u$ otras condiciones de aceptación es una cuestión trivial, no

Notre Dame Joxrnal, isid., 119-156, y tambien, del bltimo, "Truth and the Theory of Content", en H. Parret y J. Bouverease (eds.), Meaning and Underatanding (Gragter, Berlín, 1981). Vease en Gilbert Harman, "Conceptual Role Semantics", Notre Dame Journal of Formal Logie, xxiii (1982), 242-256, una teoría del papel conceptual no interna en el sentido meñalado en el texto.

- Véase Loar, op. cil., para un enunciado de algo que mería una consecuencia de esta tesis: "Dudo que en exalgier teoris del uso (cualquiers en la que el aignificado sea cuestión de reglas, procedimienton de verificación, etc.) lo que constituya el significado de una oración explicará - vinficara la asignación de nuestras condiciones de verdad preferidas - eas oración” (282-283). Las concepciones má detalladas de Loar, según las cuales esa formulación se especificaría para las creencias obmervacionales, se ofrecen en su Mind and Meaning (CUP, Cambridge, 1981), esp. Capítulo 8. 
algo que necesite argumento para cada tipo particular de contenido. ${ }^{4}$ Antes de que podamos evaluar esas afirmaciones adicionales, necesitamos conocer más acerca de la posición que excluyen: aquí me dedicaré a la elaboración adicional de una noción de una condición de aceptación y su relación con las condiciones de verdad, que esas afirmaciones adicionales excluirían.

1. Consideremos primero contenidos en los cuales un concepto observacional se predica en tiempo presente de un objeto presentado demostrativamente en la percepción: nos ocuparemos de casos en los cuales el objeto es de tal tamaño que puede, en nuestras circunstancias presentes, determinarse al percibir el objeto, caiga o no bajo el concepto observacional. Tales contenidos o pensamientos se expresarían en español mediante "Este bloque es cúbico", "Este plato es ovalado", "Esta superficie es azul"; pero la posesión de actitudes hacia esos contenidos no se restringe necesariamente a criaturas que posean lenguaje. Los conceptos se toman aquí como constituyentes de los contenidos. Por lo tanto, se conciben de tal manera que se conformen a la condición fregeana de informatividad, una versión de la condición que para Frege correspondientemente gobernaría los sentidos de las palabras predicativas: si el contenido "Un objeto es $\varphi$ si y solamente si es $\psi^{\prime \prime}$ es potencialmente informativo, entonces $\varphi$ y $\psi$ son conceptos distintos. Si no elaboramos finalmente esto, dejaremos fuera demasiados rasgos distintivos del fenómeno cognoscitivo.

Los contenidos dentro del rango escogido tienen los siguientes componentes. En el primer componente, el objeto percibido se presenta de cierta manera $W$ en la percepción; algún concepto $F$ - "bloque", "plato",

- Harman es un representante de esta segunda actitud: cf. su Conceptral Role Semantics, op. cit. 
"superficie" - se usa comúnmente al individuar el objeto presentado; y el objeto presentado mismo, digamos $x$. Así, el primer componente puede capturarse en la notación $\left[W, F()_{x}\right]$. En segundo lugar, está el concepto observacional $\varphi$. En tercero, está el tiempo, digamos $t$, dado como el presente en esos contenidos: podemos escribir este componente del contenido como [ahora ${ }_{t}$ ]. $E l$ contenido entero "Este $F$ es $\varphi$ " es $\left[W, F()_{x}\right] \wedge[\varphi] \wedge$ [ahora $a_{t}$ : aquí " $\wedge$ " designa la operación de formar el componente sobre constituyentes de contenidos que corresponden, en este caso simple, a la concatenación de expresiones lingüísticas para esos constituyentes. ${ }^{5}$

Si alguien juzga uno de esos contenidos, ¿en qué compromisos incurre al hacerlo? Al preguntar esto estoy preguntando acerca de un tipo particular de condición de aceptación. En particular, pregunto acerca del espectro de compromisos no revocables atribuibles a un pensador en virtud de su juzgar precisamente ese contenido, en oposición a compromisos en que pudo haber incurrido dadas hipótesis auxiliares mediante las cuales el pensador conecta ese contenido con otros. Porque se asocian con el contenido mismo, esos compromisos pueden denominarse también canónicos. Como una primera aproximación para el espectro de compromisos canónicos podemos decir:

(C) El espectro de compromisos canónicos de alguien que juzga un contenido en $t$ "Este bloque es cúbico" es que: para cualquier posición desde la cual se percibiera el bloque en $t$ por un percipiente que funcione mínimamente en condiciones norma-

- En lo que sigue nada depende esencialmente de que $x$ y mismas sean constituyentes del contenido (aunque bajo modos de presentación). Cualquier apareamiento de un contexto junto con algo que, aplicado al contexto, de $x$ y $t$, puede adaptarse a los propósitos que siguen. El constituyente $[\varphi]$ también podrá descomponerse aún más. Los corchetes alrededor de ' $\varphi$ ' se usan sólo por uniformidad de estilo en la representación formal. 
les externas, el bloque sería experimentado desde esa posición como cúbico, o como un objeto cúbico sería experimentado desde esa posición relativa. ${ }^{\circ}$

En una teoría más plena esta especificación de los compromisos canónicos, como la especificación para cualquier otro tipo de contenido, sería derivable de una especificación de la contribución a los compromisos canónicos hechos por los constituyentes del contenido dado. La idea que hay detrás de este primer esfuerzo es que, al percibir el bloque como cúbico, un sujeto puede confirmar que una de las muchas ejemplificaciones de la condición universalmente cuantificada exhibida es verdadera. En el contexto de las hipótesis auxiliares acerca de lo que sucede cuando se mueve, las otras ejemplificaciones pueden confirmarse inductivamente. Lo que el pensador tiene que hacer para mantener la atención sobre el objeto cuando él o el objeto se mueve, depende en parte de cómo el objeto se le presenta a él en la percepción, y en parte del tipo de objeto que es. No sólo confirma el pensador realmente en su experiencia perceptual únicamente un fragmento del espectro de compromisos; no sabe infaliblemente que ha confirmado ese fragmento, puesto que no sabe infaliblemente acerca del estado de sus mecanismos perceptuales o de sus condiciones ambientales $\mathrm{y}$, en todo caso, moverse alrededor del objeto toma tiempo. Pero dejando la in-

- Esto se aplica sólo a predicaciones observacionales monádicas de objetos presentados perceptualmente. Un tratamiento diverso se requiere incluso para algunas relaciones observacionales, particularmente cuando alguno de sus términos es el mismo sujeto que percibe. Difícilmente podría requerirse para la satisfacción de los compromiso: canónicos de un pensamiento en primera persona "estoy enfrente de esa casa (presentada perceptualmente)", que si estuviese en cualquier posición cercana diferente, aún tendría una experiencia como de mí mismo eatando enfrente de la casa. El requerimiento tendria que referirse a la relación percibida desde otra posición entre el lugar en el que estaba localizado previamente y la casa. 
falibilidad a un lado, pueden confirmarse ejemplos de este espectro de compromisos canónicos. El pensador no necesita, desde luego, tener esas especificaciones de los compromisos canónicos conscientemente en la mente, o ser capaz de formularlas. Lo que importa es que ellas describan correctamente sus compromisos. Que éstos son sus compromisos se mostrará por las circunstancias en las cuales desee revocar un juicio sobre el contenido. Supongamos que un pensador no cuestiona la normalidad de su entorno, ni la operación de sus mecanismos perceptuales: entonces el patrón de sus aceptaciones o rechazos reales y contrafácticos de "Este bloque es cúbico", en respuesta racional a sus experiencias perceptuales, puede hacer razonable atribuirle el espectro de compromiso en (C).

Dos pensadores pueden variar grandemente en cuanto a su temeridad inductiva, pero juzgar sin embargo exactamente el mismo contenido. El pensador más osado puede requerir mucho menos que el pensador más tímido en lo que concierne a la evidencia de que se satisfacen los compromisos canónicos de uno de nuestros contenidos observacionales. Pero los compromisos en que incurre, sobre una base evidencial más estrecha, son los mismos que los del pensador tímido al juzgar el mismo contenido: argumentaré que esto es lo que hace que estén juzgando el mismo contenido.

En el caso del concepto de ser cúbico, y de otros conceptos de cualidades primarias, no hay una modalidad de un único sentido que haga que la posesión de esos conceptos exija una sensibilidad específica a las percepciones en esa modalidad sensorial particular. Tanto una experiencia visual como una táctil, pueden representar algo que se tiene en la mano como cúbico, o dos bordes cercanos como paralelos, o como afilados. Lo que importa aquí es el orden relativo del operador modal y 
el cuantificador. No hay una modalidad de tal índole que, para poseer esos conceptos, sea necesaria una sensibilidad a las percepciones en esa modalidad: pero es necesario que haya percepciones en alguna modalidad tal que haya cierta sensibilidad racional de los juicios que contienen el concepto para esas percepciones, si el concepto ha de ser observacional. Mi discusión aludirá principalmente a la experiencia visual: pero, en el caso de un concepto observacional de una cualidad primaria, será suficiente para la posesión del mismo que las condiciones mencionadas para la experiencia visual se cumplan correspondientemente para las experiencias en alguna otra modalidad sensorial. A las cualidades primarias se puede acceder a través de diferentes modalidades precisamente porque son conceptos de propiedades objetivas cuya naturaleza es independiente de cualquier forma particular de experiencia sensorial.

Esta objetividad no está de hecho plenamente capturada en esa primera aproximación al espectro de compromisos canónicos. Podemos concebir algo que cambia de forma conforme un percipiente normal se mueve a su alrededor; y podemos concebir que lo hace de tal manera que desde cualquier posición el percipiente lo vea cúbico aun cuando de hecho nunca sea realmente cúbico. El espectro pleno de compromisos canónicos ofrecido en la primera aproximación puede cumplirse sin agotar los compromisos reales de alguien que juzga el contenido. Los compromisos reales excluyen el estado de cosas que se acaba de describir. No es correcto decir que este punto ya está acomodado mediante la apelación a condiciones normales en la primera aproximación. Considérese un escéptico curioso que sostiene que las cosas alrededor de nosotros frecuentemente cambian su figura conforme nos movemos alrededor de ellas. ¿Podemos realmente responderle diciendo: "Pero ésas son 
las condiciones normales en las que estamos, de manera que tu escepticismo es ininteligible"? Por el contrario, no hay ningún problema para comprender (en oposición a creer) esta hipótesis escéptica. Lo que, en el caso del concepto de ser cúbico, se ha omitido de los compromisos canónicos, es esto: la figura real del objeto en las circunstancias presentes tiene que ser la responsable, en las circunstancias contrafácticas mencionadas, de las experiencias perceptuales. Lo que sucede en las circunstancias contrafácticas en las cuales el objeto tiene una figura diferente de la que realmente posee es irrelevante para el cumplimiento de esos compromisos. El juicio de que el objeto presentado es cúbico debe ser revocado si resulta patente que la figura real no es responsable de las experiencias relevantes, aun si todos los contrafácticos en la primera aproximación son verdaderos - como podrían serlo de acuerdo con las curiosas hipótesis del escéptico. (Igualmente, si un concepto de color se predica de una superficie, alguna propiedad real de la superficie del objeto debe, en las circunstancias contrafácticas, causar las experiencias mencionadas en los compromisos canónicos.) Es importante también que, en el enunciado mejorado de los compromisos canónicos, digamos que es la figura real del objeto la que es responsable de las experiencias, en vez de decir que el responsable es el hecho de que el objeto es cúbico. El último enunciado, aunque verdadero, trivializa la conjetura; el primero no lo hace.

¿Qué es un "percipiente que funciona mínimamente" ? $¿$ Es condición necesaria para que un sujeto sea un percipiente que funciona mínimamente, el hecho de que si su experiencia representa su entorno de cierta manera, éste sea de esa manera? Pero este requerimiento de concordancia es demasiado fuerte. Alguien puede preguntar: "Yo sé que mis mecanismos perceptuales funcionan bien 
y que la iluminación es normal, pero las cosas que veo cuadradas, ¿no serán acaso realmente no cuadradas?". Esta es una especulación coherente. Una explicación filosófica de un percipiente que funciona mínimamente debe dar acomodo al hecho, $\tan$ familiar a los psicólogos y artistas, de que, por ejemplo, aun con el comportamiento normal de la luz, muchos diferentes trapezoides irregulares pueden causar la experiencia visual de algo cuadrado en el entorno. Estas figuras diferentes tienen en común el causar el mismo patrón de estimulación retiniana. Si impusiéramos el requerimiento de concordancia a los percipientes que funcionan mínimamente, nuestro dubitativo sujeto estaría considerando algo que es a priori incoherente, lo que no hace.

Más que imponer el requerimiento de concordancia, podemos proceder de la siguiente manera. Una descripción de las cosas en el entorno de un sujeto está en la clase de proyección de un patrón dado de estimulación retiniana sólo en el caso de que la existencia de las cosas en el entorno que caen bajo esa descripción pudiera, con el comportamiento normal de la luz, ser parte de la explicación causal de la ocurrencia de un ejemplo de ese patrón de estimulación retiniana. Una descripción de la forma "Una puerta de tal y cual figura, color y textura, con tal y cual orientación y distancia relativa al sujeto", está en la clase de proyección del patrón de estimulación retiniana que normalmente se recibe cuando se mira hacia esa puerta. Esa misma clase tendrá también como miembro la descripción de una fachada cuidadosamente pintada desde un ángulo muy diferente que no contiene ninguna puerta real o algún segmento saliente que tenga figura de puerta, pero que con el comportamiento normal de la luz produce el mismo patrón de estimulación retiniana. Con esta noción, podemos decir entonces que un sujeto es un percipiente que funciona 
mínimamente sólo si se cumplen dos condiciones cuando tiene una experiencia perceptual en circunstancias externas normales: (a) la descripción bajo la cual la experiencia representa las cosas en el entorno está en la clase de proyección del patrón de estimulación retiniana que causa la experiencia; y (b) se dan relaciones apropiadas de explicación causal entre la ocurrencia de ese patrón y la experiencia (los detalles no importan aquí). Un percipiente que funciona mínimamente no será necesariamente muy eficiente - podría ser mucho menos eficiente que los humanos- pero lo que sigue es verdad respecto de todo percipiente que funciona mínimamente. Al ver un objeto desde posiciones diferentes, obtiene una clase de descripciones de lo que está en su entorno, las cuales están en las clases de proyección de todos los patrones sucesivos de estimulación retiniana. Este será en general un conjunto mucho más reducido que la clase de proyección de cualquier patrón semejante. En particular, una descripción del entorno que contiene un trapezoide irregular no estará en la clase de proyección de todos los patrones sucesivos cuando un cuadrado se ve desde diferentes ángulos. Ésta es la razón por la que todavía importan las visiones desde diferentes posiciones. De manera similar, las notas de los objetos que causalmente no son operativas en la producción de experiencias cuando el sujeto está en un sitio, pueden cobrar significación cuando éste se mueve. ${ }^{7}$

En el enunciado (C) de los compromisos canónicos, debe entenderse que la condición entera universalmente cuantificada que comienza con "Para cualquier posición..." cae dentro del alcance del compromiso del pensador al juzgar "Este bloque es cúbico". Supongamos

7 Estos ejemplos y observaciones modifican sustancialmente las tesis acerca de la explicación diferencial y la definición de percepción en mi Holiatic Explanation (Clarendon Presa, Oxford, 1979), Capítulo 2. 
que el pensador continúa creyendo el contenido "Este bloque es cúbico" ante una de sus experiencias del bloque que lo representa curvo. Entonces, él se comprometerá a creer que o bien las condiciones externas no son normales o sus mecanismos perceptuales no están funcionando apropiadamente. Además, no necesita ser capaz de formular esto explícitamente: que ha incurrido en esos compromisos se mostrará en las maneras reales y contrafácticas en que trata de hacer coherentes sus juicios.

Hasta aquí los preliminares. ¿Qué importancia tiene esto para la relación entre condiciones de aceptación y condiciones de verdad? Para los contenidos del tipo que nos ocupan, sostengo:

(S.Obs) Para cualquier contenido tal, si todos sus compromisos canónicos se cumplen, entonces es verdadero.

(S.Obs) se llama así porque da una condición suficiente para la verdad de uno de nuestros contenidos observacionales. También sostengo:

(N.Obs) Para cualquier contenido tal, si es verdadero, entonces todos sus compromisos canónicos se cumplen.

Se llama así porque da una condición necesaria. Si (S.Obs) y (N.Obs) son verdaderos, entonces para esta clase restringida de contenidos, ciertas condiciones de aceptación - los compromisos canónicos- determinan las condiciones de verdad. (S.Obs) debiera ser el caso más controvertido. Para cualquier concepto observacional particular $\varphi$, se argumentaría para ejemplificaciones de (S.Obs) que lo contienen por contraposición: recuérdese que aún estamos interesados aquí sólo en 
conceptos $\phi$ tales que, como son las cosas, puede determinarse, mediante la percepción de algo, si es $\phi$. El argumento para (S.Obs) es entonces que si las condiciones externas son normales, cualquier cosa que no es $\varphi$ tiene en realidad una propiedad que, desde alguna posición, causará que no sea experimentada por un percipiente que funcione mínimamente como lo sería una cosa observablemente $\varphi .^{8}$ Como se prometió, este argumento no procede a partir de una identificación general anterior de la verdad con el cumplimiento ideal de condiciones evidenciales, sino que establece puntos específicos para el tipo de contenido en cuestión. El argumento es, por ejemplo, totalmente inaplicable a conceptos que no poseen las conexiones con experiencias perceptuales distintivas de las nociones observacionales. También sería inaplicable a todo modo natural de pensar que contenga algún componente perceptual, pero que requiera también cierta constitución interna o teórica de sus ejemplificaciones. ${ }^{9}$

- También podría construirse un argumento más general relevante para las instancias de $(S)$ en relación con conceptos de forma partiendo de un teorema impresionante de Ullman: véase au artículo "The Interpretation of Structure from Motion", Proc. Royal. Soc. of London, B 203 (1979), 406-426. Ahl muestra que tres visiones ortográficas diatintas de cuatro puntos no-coplanares sobre un objeto rigido son suficientes para determinar su forma tridimentional (hasta una reflexión). Un sistema perceptual eficiente explotará cate hecho: no debemos esperar que se necesite un vasto número de visiones para determinar la forma tridimensional.

- No hemos establecido que las condiciones de aceptación determinen las condiciones de verdad ni para todos los contenidos de nuestra forms observacional elegida: porque estuvimos operando bajo la reatricción de que el objeto presentado perceptualmente es uno para el que la aplicabilidad del concepto observacional puede determinarse perceptualmente. La restricción es genuina: considerese “esta eatrella es esferica". Para establecer la conjetura para todos los contenidos de este tipo se requerirán argumentos de un tipo muy distinto, que traten con inferencias teóricas de la forma (por ejemplo) en lo muy grande o lo muy pequeño, las cualea no juegan ningún papel on los casos del texto. 
El argumento que se acaba de dar toca tres nociones que conciernen al contenido sin elucidarlas. Da por seguro el contenido conceptual de la experiencia. También emplea la idea de que es un objeto externo, y no algún otro ser el que se presenta demostrativamente y acerca del cual se piensa. Por último, simplemente usa la noción de compromisos que son satisfechos, esto es, verdaderos. Seguramente todas estas nociones necesitan más elucidación, $y$ todas la necesitan apelando a las relaciones entre los estados del pensador y las cosas externas. Así la explicación de la relación entre la aceptación y la verdad que dan (S.Obs) y (N.Obs) es parcial. Sin embargo, es importante que podamos dar esa explicación, porque al darla satisfacemos la necesidad de tener una explicación que posea lo que podemos llamar determinación interna. Porque sería problemático si, incluso cuando nos está permitido tomar lo que he tomado sin merecerlo, las condiciones de verdad de estos contenidos simples sobrepasaran sus condiciones de aceptación.

Algunas veces consideraremos como verdadero un contenido como: "Si estuviese en el cuarto de junto, aún sería el caso que aquella mesa (presente perceptualmente) es rectangular" $\left(\left[W\right.\right.$, Mesa ()$\left._{x}\right] \wedge\langle$ ser rectangular $\rangle$ $\wedge\left[\right.$ ahora $\left.\left._{t}\right]\right)$. Esto no es equivalente a: "Si estuviese en el cuarto de junto un contenido de la forma $\left[W, \operatorname{Mesa}()_{\eta}\right]$ $\wedge$ (ser rectangular $\rangle \wedge$ [ahora $t$ juzgado por mí ahí sería verdadero". Así pues, ¿cómo explicamos el papel de nuestros contenidos observacionales en tales incrustaciones? En general, para cada contenido de la forma $\left[W, F()_{x}\right] \wedge[\varphi] \wedge\left[\right.$ ahora $\left._{t}\right]$ hay una proposición correspondiente $\langle x, P, t\rangle$ que consiste en el objeto percibido $x$, la propiedad $P$ presentada por el concepto observacional $\varphi$ en el contenido, y el tiempo presentado (una proposición russelliana). Para los propósitos de evaluar estos contrafácticos, la verdad del contenido con res- 
pecto a circunstancias no-actuales requiere que el objeto $x$ tenga la propiedad $P$ en $t$, esto es, que su proposición correspondiente sea verdadera. A menos que haya alguna dificultad acerca de qué objeto y qué propiedad se presentan así, los principios que dan los compromisos canónicos de estos contenidos serán suficientes, si usamos la noción de la proposición correspondiente, para la determinación de lo que tiene que ser el caso para que el contenido sea verdadero en cualquier circunstancia arbitraria.

Si la conjunción de (S.Obs) y (N.Obs) nos permite decir que los compromisos canónicos determinan las condiciones de verdad, difícilmente podemos negar que en el mismo sentido las condiciones de verdad, aquí, determinan los compromisos canónicos. Pero esto no debe ser malentendido. (S.Obs) dice que el cumplimiento de los compromisos asegura el cumplimiento de las condiciones de verdad, y (N.Obs) dice la conversa: ni (S.Obs) ni (N.Obs) dicen nada específicamente acerca de singularidad. En particular, (N.Obs) no garantiza que con cada condición de verdad, si (para los propósitos de este párrafo solamente) eso se toma en estos ejemplos como dado por el triplo ordenado de un objeto, una propiedad y el tiempo, haya asociado un patrón único de compromisos canónicos. (N.Obs) asegura solamente esto: si se da la condición de verdad para un contenido, el espectro de compromisos para ese contenido se satisface. Esto es consistente con la no-existencia de una ruta de regreso de la referencia al sentido: porque puede ser informativo decir de dos espectros de compromisos que uno es satisfecho si y sólo si el otro lo es. Así que aún puede haber una única asociación determinante-de-contenido de un espectro de compromisos canónicos con un contenido dado. Así, supongamos que podemos a la vez sentir calor y verlo con visión infrarroja. Si los términos "calor ${ }_{1}$ " 
y "calor 2 " se introducen para las propiedades físicas presentadas de estas dos maneras, el pensamiento de que algo está caliente 1 si y sólo si está caliente 2 puede ser informativo. Correspondientemente, considérense los dos pensamientos de que un objeto, presentado de una manera dada, está caliente ${ }_{1}$ y de que está caliente ${ }_{2}$. Los compromisos canónicos del primer pensamiento se satisfacen todos si y sólo si se satisfacen los compromisos canónicos del segundo pensamiento: pero ésta es una verdad a posteriori. Los compromisos canónicos efectivamente serán determinados únicamente por un objeto, propiedad y tiempo, bajo modos adecuados de presentación. Pero según la concepción que subyace en este trabajo, esto no es una sorpresa: porque la concepción es que puede darse una teoría sustantiva del sentido apelando a las condiciones de aceptación, de las cuales una especificación de los compromisos canónicos no es sino un caso especial. ${ }^{10}$

Cuando el componente predicativo de uno de nuestros contenidos observacionales es un concepto de cualidad primaria, el contenido es tal que puede ser escuetamente verdadero, en el sentido de Michael Dummett ${ }^{11}$ adaptado a contenidos: para él, un enunciado escuetamente verdadero es uno para el que no hay una respuesta notrivial a la pregunta “¿Qué lo hace verdadero?”. Dummett también ha dicho que nuestro modelo para el conocimiento de lo que significa que un enunciado capaz de

10 Otra cuestión genuina sobre la cual trabajar es si hay una unicidad correspondiente en el caso de (S.Obs): ésta es la cuestión de la indeterminación de la asociación de las condiciones de verdad con las condiciones de aceptación. Una concepción escéptica sobre la determinación, basada en una noción distinta de condiciones evidenciales y del papel conceptual, puede verse en Schiffer, "Truth and the Theory of Content", op. cit.

11 Véase su "What is a Theory of Meaning? (II)", en Gareth Evans y John McDowell (eds.), Truth and Meaning (Clarendon Press, Oxford, 1976), 89 y ss. 
ser escuetamente verdadero sea verdadero, es la habilidad de usarlo para dar un reporte de observación. ${ }^{12}$ Esto puede necesitar especificación si admitimos que los contenidos acerca de las entidades postuladas por la ciencia física sean escuetamente verdaderos: pero para contenidos observacionales, lo que he argumentado hasta ahora apoya la tesis de Dummett. El punto no es que siempre que, por ejemplo, un sujeto que funcione mínimamente tenga una experiencia de algo cúbico en circunstancias externas normales, su experiencia será causada por el ser cúbico del objeto percibido: ya hemos visto que esto es falso. Lo que si es verdadero es que la única propiedad del objeto presentado que explicaría el que apareciera cúbico (o como aparecería un objeto cúbico) desde todas las posiciones relativas es que sea cúbico. Esta característica puede ser única para los contenidos observacionales de cualidades-primarias; y esto ayuda a explicar cómo podemos comprender un rango de contenidos que pueden ser escuetamente verdaderos.

Dos pensadores diferentes pueden confirmar, a su satisfacción, que los compromisos canónicos de un contenido observacional dado están satisfechos. Pero ya que cada uno confirma que están satisfechos en el caso de sus propias experiencias, surge la pregunta de cómo ellos pueden estar juzgando exactamente el mismo contenido. ¿Por qué, por ejemplo, no están juzgando más bien contenidos del mismo tipo? La determinación de las condiciones de verdad por las condiciones de aceptación nos ayuda a contestar esta pregunta. La condición de verdad, que concierne a las propiedades reales, causalmente operativas del objeto presentado, no es relativa a la persona. Si los compromisos en (C) son satisfechos con respecto a la experiencia de un pensador cualquiera, 
entonces se cumplen con respecto a la de cualquier otro pensador. De aquí que no importe la ambigüedad en (C) de la frase "Un percipiente que funcione mínimamente". Al argüir esto, estamos argumentando en favor del cumplimiento de una condición de adecuación sobre una explicación del contenido en función de condiciones de aceptación que siempre debe satisfacerse, a saber: si las condiciones de aceptación determinantes del contenido son para un contenido de alguna manera relativas al pensador, mientras que su condición de verdad no lo es, debemos ser capaces de establecer que, si las condiciones de aceptación se cumplen para un pensador cualquiera, entonces se cumplirán para cualquier otro pensador.

Habría otras varias consecuencias de la determinación de las condiciones de verdad por los compromisos canónicos. Una sería que un teórico que aspira a individuar el contenido mediante ciertas condiciones de aceptación (incluyendo los compromisos canónicos) podría aceptar consistentemente los argumentos de Dummett de que un contenido (un pensamiento) es esencialmente algo que, junto con el mundo, determina un valor de verdad. ${ }^{13}$ Los argumentos de Dummett sugieren que es una condición de adecuación sobre una teoría del sentido que trate a los contenidos (pensamientos) como determinantes esenciales de valores de verdad, dada la manera en la que es el mundo. Si las condiciones de aceptación determinan las condiciones de verdad, entonces una teoría que usa las condiciones de aceptación para individuar el contenido puede satisfacer esta condición de adecuación. Otra consecuencia se refiere a la tesis de que llegar a entender un lenguaje, $o$ a aprehender ciertos contenidos, es en parte cuestión de adquirir una

18 Michael Dummett, The Interpretation of Frege's Philosophy (Duckworth, Londres, 1981), 47. 
sensibilidad racional adecuada para las condiciones de aceptación (que pueden incluir condiciones evidenciales). Esta tesis no necesita competir, sino que de hecho en realidad implicaría la tesis de que estos logros pueden igualmente describirse como la adquisición de la comprensión, de una manera particular, de las condiciones de verdad de los contenidos. ${ }^{14}$ Esto le da importancia a la cuestión de si alguna forma del modelo presente puede aplicarse a otros tipos de contenido.

2. Los contenidos observacionales que hemos estado considerando forman un caso muy especial. Pero la relación entre la verdad y las condiciones de aceptación en este caso es una instancia de una forma más general. La forma más general es que para cada contenido hay un espectro de compromisos tales que el contenido es verdadero si y sólosi se satisfacen todos esos compromisos. En el caso observacional estos compromisos comprendían percepciones que representan al mundo en cierta manera de ser, manera conectada sistemáticamente con el concepto principal del contenido: pero, para otros contenidos, la operación correlacionante que determina los compromisos a partir del contenido puede ser diferente. Una dirección en la que podríamos generalizar el modelo inicial de las relaciones entre verdad y aceptación para un nuevo rango no-observacional de contenidos, sería esta:

Hay alguna operación $C$ tal que para cada contenido $p$ en el rango:

(a) $C(p)$ da los compromisos canónicos de $p$ y

(b) $p$ es verdadera si y sólo si se dan todas las condiciones en $C(p)$.

14 Para una concepción radicalmente opuesta, véase Brian Loar, "Conceptual Role and Truth Conditions", 276 y ss., espocialmente la p. 4. 
Mientras que un rango de contenidos puede individuarse por sus compromisos canónicos, otro rango puede individuarse por sus condiciones canónicamente comprometedoras por sus fundamentos canónicos más que por sus consecuencias canónicas. Para un rango de contenidos individuados por sus fundamentos canónicos, podemos decir:

Hay alguna operación $G$ tal que para cada contenido $p$ en el rango:

(a) $G(p)$ da los fundamentos canónicos de $p, y$

(b) $p$ es verdadera si y sólo si se da alguna condición en $G(p)$.

Que un contenido tiene ciertos fundamentos canónicos se mostrará en las condiciones que un pensador considera suficientes para sostenerlo.

Una versión más refinada de cada uno de estos modelos podría acomodar alguna dependencia, en la determinación de la condición de verdad, respecto de las actitudes del pensador individual. Con tal que la naturaleza de esta dependencia sea dada uniformemente a partir del contenido $p$, esto no necesita en ninguna manera fundamental volver a las condiciones de verdad relativas a la persona. En una versión refinada del modelo de compromisos, por ejemplo, habría una operación adicional $B$ :

Hay operaciones $C$ y $B$ tales que para cada contenido $p$ en el rango y para cada pensador $x$ :

(a) $C(p)$ da los compromisos canónicos de $p, y$

(b) $p$ es verdadera si y sólo si se dan todas las condiciones en $C(p)$ y todas las condiciones en $B(p, x)$.

Daré un ejemplo de este modelo más tarde. Uno puede entrever variaciones mucho menos restrictivas sobre este tema. 
Nuestra pregunta ahora es: ¿puede alguna forma de uno de esos modelos esquemáticos ser aplicada a contenidos que son cuantificaciones objetuales universales "Todos los $F$ son $G$ " $(\forall x(F x, G x))$ ? $^{15}$

En el espíritu de Ramsey podríamos tratar de construir una teoría alrededor de la idea de que alguien que cree que todas las cosas $F$ son $G$ está dispuesto, teniendo como premisa el pensamiento de que $m$ es $F$, a inferir que $m$ es $G .{ }^{16}$ Una teoría construida en este espíritu debe resolver dos problemas. El primero es que la teoría debe explicar cómo es que los contenidos cuantificacionales encajan dentro de operadores más complejos. Sobre este primer problema, el teórico puede decir lo siguiente. Supongamos que la disposición inferencial de alguien que cree que todos $\operatorname{los} F$ son $G$ ha sido caracterizada satisfactoriamente. Podemos preguntar qué condición tiene que mantenerse en el mundo para que el ejercicio de esta disposición nunca lleve a una creencia falsa. Ésta será la condición de verdad resultante para el contenido; y esta condición de verdad puede ser suministro, en los casos extensionales, para que otros operadores sean aplicados cuando una cuantificación es encajada en contenidos más complejos. ${ }^{17}$ Ésta es una adaptación de una táctica ya adoptada en el caso observacional. El segundo problema es que, tal como se encuentra la teoría, la disposición que propone también

15 Las razones para preferir la notación binaria dada en el paréntesis a la estándar $\forall x(F x \rightarrow G x)^{»}$ están resumidas en Martín Davies, Meaning, Quantification, Necessity (Routledge and Kegan Paul, Londres, 1981), 123-136.

$10(x) \varphi x$ expresa una inferencia que estamos preparados para hacer en cualquier momento: F. P. Ramsey, The Foundations of Mathematice, 240. 17 Aqui el térico se aparta de Ramsey, quien parece haber sostenido que las oraciones cuantificadas no expresan proposiciones exactamente en el mismo sentido en que lo hacen sus inst ancias puramente singulares: cf. The Foundations of Mathematics, 240. 
la poseería un pensador cuya cuantificación "Todos los $F$ son $G^{\text {n }}$ tenga la condición de verdad de que todos los objetós de los que él tiene o tendrá modos de presentación de cualquier tipo en su repertorio, son $G$. El teórico tiene que ofrecer una explicación que determine únicamente la cuantificación objetiva sobre todos los objetos de la clase dada.

Las explicaciones de la cuantificación raramente van dirigidas a la cuestión de qué hace que el pensamiento de un sujeto sea un pensamiento que contenga cuantificación objecional no-restringida, en lugar de algún análogo para el pensamiento de cuantificación sustitucional. Gareth Evans, por ejemplo, en una discusión iluminadora, escribió: "La proposición de que algún $F$ es $G$ se concibe de tal modo que llegaría a ser verdadera por la verdad de alguna proposición de la forma ' $\delta$ es $G\urcorner$, donde $\delta$ es una Idea fundamental de un $F$ ' ${ }^{18}$ No es objeción a esto que la frase "Alguna proposición de la forma..." esté dentro del alcance de "Se concibe de tal modo que...": Evans no estaba tratando de dar un análisis eliminativo de la aprehensión de la cuantificación existencial, sino un principio que la describiese. Pero no necesita haber una Idea o modo de presentación $\delta$ en el repertorio presente o futuro del pensador que verifique una cuantificación existencial verdadera que el pensador juzga. ${ }^{19} \mathrm{Si}$ tenemos una perplejidad acerca de la naturaleza del dominio de la cuantificación norestringida sobre objetos, el enunciado de que tal cuantificación se concibe como verdadera en virtud de una condición que envuelve cuantificación no-restringida sobre Ideas fundamentales de esos objetos no debería eliminar toda nuestra perplejidad. Podríamos estar ten-

The Varieties of Reference (Clarendon Press, Oxford, 1982), 108.

Como observó Evans: ibid., pp. 108-109. 
tados a decir que estamos considerando un pensador ideal tal que para cada $F$, hay alguna idea fundamental o modo de presentación de ella en su repertorio. Pero entonces debemos explicar cómo nuestro pensamiento real, no-ideal, alcanza las condiciones de verdad de los pensamientos del pensador ideal; y al hacerlo así debemos también explicar nuestra aprehensión del pensamiento "Para cada $F$ hay alguna Idea fundamental de ella en el repertorio del pensador ideal".

Este problema, por supuesto, no es específico de los cuantificadores universal y existencial. El problema surge para cualquier operador de segundo-nivel cuyas condiciones de verdad envuelven ineludiblemente el rango completo de objetos que caen bajo algún concepto: surge igualmente para el operador de la descripción definida y para predicados de segundo-nivel como 'raro', tal como ocurre en "Los albinos son raros". Lo que debemos dar es una elucidación del fenómeno que Dummett caracteriza diciendo: "Entendemos el enunciado cuantificado universalmente porque tenemos, por decirlo así, una aprehensión general de la totalidad que constituye el dominio de la cuantificación - por decirlo así, lo contemplamos en el pensamiento como un todo..." 20

Buscamos una explicación de la aprehensión de la cuantificación universal objetual en términos de compromisos canónicos. La explicación debe determinar las condiciones de verdad objetuales, y explicar así el hecho notable de que los humanos somos capaces de aprehender un contenido que requiere para su verdad algo acerca de todos los objetos de una clase dada. Ahora bien, ciertamente habrá diferencias, accesibles a un intérprete radical, entre alguien cuyas cuantificaciones son objetuales y alguien cuyas cuantificaciones son sus-

20 Prege: Philosophy of Language (Duckworth, Londres, 2a. ed., 1981), 617. 
titucionales. Las condiciones empíricas que hacen razonable juzgar una cuantificación universal objetual son mucho más exigentes que las que hacen razonable juzgar una cuantificación sustitucional. ${ }^{21}$ En algunas definiciones de validez, también habrá diferencias en la lógica a la que se conforman los dos cuantificadores. ${ }^{22}$ Pero estos puntos indiscutibles no nos dan totalmente lo que estamos buscando: o llegan demasiado tarde o suscitan los mismos problemas. Estamos pidiendo cierta forma de tratamiento que explique cómo es que un contenido puede abarcar a todos los objetos. Dado que sí puede, el que lo haga tendrá las consecuencias mencionadas que lo diferencian de la cuantificación sustitucional. Pero estas consecuencias particulares no constituyen una explicación de qué es lo que hace que el contenido tenga

21 Asumo que todos los términos en la clase de sustitución denotan. Si no lo hacen, aún habrá otras diferencias empíricas entre los cuantificadores interpretados de cada una de las dos formas.

22 Saul Kripke observa que si definimos la validez para las fórmulas sustitucionales de una manera que tome como rango de clases de sustitución conjuntos arbitrarios de expresiones de la categoría apropiada, entonces coinciden la validez sustitucional y la validez objetual clásica: una fórmula es sustitucionalmente válida si y sólo si es clásicamente válida. (“Is There a Problem about Substitutional Quantification?", en Evans y McDowell (eds.), Truth and Meaning, p. 336.) Esta no es, sin embargo, la única definición de validez posible, y también hay maneras de elaborar la idea de que algo que sea aceptado como una ley se vincule a otra definición de valides. En particular, como observa Davies, si las clases de sustitución sobre las que se cuantifica en la definición de validez se restringen a las que contienen expresiones que ya están en el lenguaje, es válida una clase más amplia de fórmulas ("A Note on Substitutional Quantification", Nous, xiv (1980), 619-622). En un lenguaje cuyas únicas constantes individuales son $a$ y $b$, y cuyos únicos factores formadores de términos son $f$ y $g$, cualquier instancia del esquema $[F a \& F b \& \forall x F(f(x)) \& \forall x F(g(x))] \rightarrow \forall x F x$ es válida bajo la definición alterada. Un pensador reflexivo que está usando la cuantificación sustitucional aceptará todas las instancias de este esquema; un pensador que esté usando la cuantificación objetual no lo hará. También hay diferencias en la relación de las consecuencias lógicas en la definición alterada de validez. (J. M. Dunn y N. D. Belnap, "The Substitution Interpretation of the Quantifiers", Nots, ii (1968), 177.) 
como rango todos los objetos: ciertamente no se ha mostrado que ellas determinen la condición de verdad.

Por el otro lado, algún procedimiento de interpretación radical ideal para identificar la cuantificación universal objetual específicamente, en lugar de aquellas características particulares que diferencian la cuantificación objetual de la sustitucional, podría proponerse para proveer lo que buscamos. El procedimiento ideal especificaría, al nivel de los hechos accesibles al intérprete radical, lo que tiene que ser el caso para que alguien signifique la cuantificación objetual universal mediante algo. Si nuestra conjetura inicial es correcta, desde luego es razonable esperar que un procedimiento de interpretación radical ideal para tal cuantificación y una explicación de sus compromisos o fundamentos canónicos serán equivalentes: son explicaciones desde diferentes perspectivas, la interna y la externa, de qué es lo que hace que un pensador esté juzgando un contenido y no otro. Pero aún no tenemos tal procedimiento de interpretación radical -en efecto, esto es, desde una perspectiva diferente, lo que estoy tratando de proveer.

Inicialmente conf inamos nuestra atención a la cuantificación no-restringida sobre objetos físicos que existen en el tiempo presente. Cada objeto tal es individuado por su localización actual y su tipo. Si podemos explicar la cuantificación sobre todos los lugares podemos explicar la cuantificación sobre todos esos objetos: para que todos los $F$ sean $G$ tiene que ser que para todo lugar el $F$ (si hay alguno) en ese lugar es $G$.

¿Cuál es nuestra concepción del rango de variables no-restringidas sobre lugares? Sugiero que está gobernada, inter alia, por dos principios: (a) Es cerrado bajo funciones tales como las dadas por frases de la forma "El lugar que tiene la relación espacial $R$ con el lugar $\pi$, cuando estas funciones se aplican a lugares que ya 
están en el dominio. Por supuesto que en el caso de algunas relaciones $R$, no habrá un lugar que tenga la relación $R$ con un lugar dado anteriormente en el dominio - por ejemplo, si el universo es finito- y tener $R$ con $\pi$ implica la relación de estar de $\pi$ a una distancia mayor que cierta distancia. Pero si hay un lugar tal, está en el rango de variables no-restringidas sobre lugares. (b) Entender la cuantificación no-restringida sobre lugares no consiste en la existencia de una lista finita de relaciones respecto de las cuales el que comprende aprecia que el rango de lugares está cerrado justo bajo aquellas relaciones. Más bien, esta comprensión es abierta: para cualquier relación espacial nueva que se le presenta, el que comprende considerará cerrado el rango de lugares bajo aquellas relaciones. Esto permite una fineza arbitraria de la discriminación de regiones más pequeñas, y la discriminación de distintos lugares a grandes distancias. ${ }^{23}$

De acuerdo con lo que llamaremos la Explicación Revisada, juzgar que todos los $F$ (físicos, que existen actualmente) son $G$ es comprometerse a juzgar, si se plantea la cuestión, cualquier instancia del esquema (1):

as Esto no debería necesariamente equipararse con el rango como indefinidamente extendible en el sentido de Dummett. (Un concepto es indefinidamente extendible si " para cualquier caracterisación definida de 6 l, hay una extensión natural de esa caracterización que origina un concepto más inclusivo": Truth and Other Enigmos, Duckworth, Londres, 1978, p. 195 y ss.) Si se nos permite usar los números reales en la especificación de las relacionea espaciales, habrá caracterisacionea del rango de lugares que serán máximamente inclusivas. Sin embargo, puede haber rasones filosoficas para sostener que la totalidad de los números reales no puede explicarse más que usando alguna noción indefinidamente extendible - quisé “concepto verdadero - falso de los números naturales". Asl sigue en pie la tesis de que el rango de la cuantificación irrestricta sobre lugares es o bien indefinidamente extendible o tiene que elucidarse usando alguna noción indefinidamente extendíble. 
(1) Si hay un $F$ en el lugar (si hay alguno) que tenga $R$ con $\pi$, es $G^{24}$

Aquí cualquier término que remplace a ' $\pi$ ' denota algún lugar que ya se piensa que está en el dominio de lugares. La disposición para juzgar en cuestión puede describirse como abierta, en un sentido que corresponde con el sentido en que es abierta la concepción del rango de relaciones bajo el cual el dominio de lugares es cerrado. O sea, para que un pensador juzgue ahora que todos los $F$ son $G$, es suficiente que juzgue ahora algo que lo comprometa precisamente, cuando se le presenta lo que él toma como una nueva relación espacial, a aceptar la instancia correspondiente de (1). Él no necesita saber ahora acerca de todas esas relaciones. La frase "Lo que él toma como" importa aquí. Un pensador puede creer que hay otras relaciones espaciales además de aquellas que hay realmente: sin irracionalidad, puede estar dispuesto a juzgar cualquier instancia de (1) que involucre una relación espacial real, sin juzgar que todos los $F$ son $G$.

Inversamente, si él cree que algo que es una relación espacial genuina no es tal cosa, puede juzgar correspondientemente la cuantificación universal sin tener la disposición con respecto a todo lo que es realmente una relación espacial. ${ }^{25}$

24 En el caso especial de generalizaciones de la forma "Todos los lugares son $G$ ", (1) deberfa remplazarse por "El lugar (ai lo hay) que tiene la relación $R$ con $\pi$ es $G^{\text {”. }}$.

28 Un pensador puede jusgar consistentemente que todos los $F$ son $G$ y que $r i$ hay un $F$ en tal o cual región, entonces no es $G$. Pero esto no contradice la explicación construida alrededor de (1). En la explicación revisada, no hay inconsistencia ni en este pensador ni en nuestra descripción de él. En la explicación revisada él se compromete a creer que "ai hay un $F$ en tal o cual región, entonces es $G^{\infty}$ y, a la

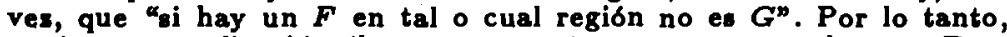
según eata explicación él se compromete a creer que no hay un $F$ en tal o cual región; y en verdad el esta comprometido a esto. 
La Explicación Revisada conserva una característica atractiva de la teoría original de Ramsey. Ofrece una explicación de por qué hay tal brecha entre juzgar que $\forall x(F x, G x)$ y juzgar que $G a \& G b \& \ldots \& G t$, donde $a . . t$ son de hecho todas las $F$ que hay. Aceptar esa última conjunción de ninguna manera es tener la disposición general e indefinidamente extensible que la Explicación Revisada exige para que uno juzgue la generalización universal. En verdad, no hay modo de presentación singular particular tal que un pensador debe, en virtud de juzgar una generalización universal, tenerlo ya en su repertorio conceptual; esto es como debe ser, y es una segunda virtud de cualquier teoría neo-ramseyana.

Si todos los compromisos canónicos, de acuerdo con la Explicación Revisada, para "Todos los $F$ son $G$ " se cumplen, ¿debe entonces ser verdad que todos los $F$ son $G$ ? No totalmente: porque los compromisos canónicos se referían a las relaciones espaciales reconocidas, las cuales pueden divergir de las relaciones espaciales reales. Todo lo que podemos decir es que si todos aquellos compromisos canónicos se satisfacen, todos los $F$ que el pensador cree que existen serán $G$. Pero permítasenos recordar la versión refinada del modelo de compromisos, la cual contenía una cláusula acerca de $B(p)$, una operación que puede referirse a las actitudes del pensador individual. Es verdad que si todos los compromisos canónicos para "Todos los $F$ son $G$ " se cumplen, $y$ el pensador está dispuesto a reconocer una relación como una relación espacial sólo en caso de que realmente sea una relación espacial, entonces todos los $F$ serán $G$. Ésta es la instancia prometida de la versión refinada del modelo.

Estos puntos presuponen que cada lugar dentro del rango de los cuantificadores del pensador está individuado por alguna extensión de los recursos conceptua- 
les del pensador para especificar relaciones espaciales. Estas extensiones incluirán la aplicación de las matemáticas al mundo real. Si alguien cree que las cuantificaciones de un sujeto a veces incluyen en su rango objetos no capturables de esta manera, podemos preguntarle qué características del pensamiento de ese sujeto ponen de manifiesto este hecho. Si puede citar tales características, la estrategia de alguien que proponga la Explicación Revisada será adaptar su teoría para acomodar aquellas características. Si no puede citarse ninguna característica semejante, seguirá escéptico respecto de que todos los objetos que debieran ser capturados permanezcan no-capturados por su explicación.

¿He estado haciendo trampa? En dos puntos he usado operadores objetuales de segundo-nivel al dar un contenido cuya aprehensión por parte del pensador presupuse cuando explicaba los compromisos de la cuantificación universal. Primero saqué provecho de la equivalencia, para predicados de objetos materiales, de "Todos los $F$ son $G$ " con "Para todo lugar, el $F$ (si lo hay) en ese lugar es $G$ ". Luego también está la ocurrencia de "Hay..." en (1). Pero esta acusación de trampa presupone que la empresa de la que me he ocupado tiene aspiraciones reductivas de un tipo que de hecho no necesita poseer. He estado tratando de mostrar cómo es que los compromisos canónicos de una cuantificación universal determinan iluminadoramente su condición de verdad clásica. Esto sigue siendo una meta razonable incluso en el contexto de una explicación de la cuantificación universal en la que tal cuantificación se use dentro del alcance de las actitudes del pensador cuya aprehensión de la noción está en cuestión. En tal contexto, la explicación presente está en verdad incompleta. Considérese la propiedad de un operador de segundo-nivel seleccionada como sigue: uno toma la conjunción de todos los compromisos 
canónicos de una cuantificación universal, y remplaza todas las ocurrencias del cuantificador universal dentro de los contextos de actitud proposicional por una variable $V$ representada apropiadamente; si el resultado es __ $V \_$la propiedad con la que nos ocuparemos es seleccionada por el término $\lambda V\left[\ldots V_{\ldots}\right]$, que es una propiedad de propiedades de segundo-nivel. Ahora la explicación es incompleta porque uno debería adicionalmente mostrar una de dos cosas que no he mostrado: - bien que la cuantificación universal es el único operador con la propiedad $\lambda V\left[\_V_{-}\right]$, o bien que hay restricciones adicionales para la cuantificación universal y que éstas, junto con la propiedad $\lambda V[\ldots V$ son conjuntamente poseídas sólo por la cuantificación universal. Sin embargo, en ambos casos la explicación en términos de compromisos puede ser una parte esencial de una explicación del dominio de la cuantificación universal.

La Explicación Revisada puede contrastarse con lo que podemos llamar teorías de relocalización. Las teorías de relocalización tratan de explicar la aceptación de $\forall x(F x, G x)$ en términos de condicionales que el pensador acepta realmente acerca de las propiedades de los $F$ que encontraría en otros lugares. Supongamos que el teórico de la relocalización dice creer que "Todos los $F$ son $G^{\prime}$ en tiempo presente es aceptar condiciones tales como (2):

(2) Si viajo hacia el norte durante un día, y ahí aprendo algo de la forma "el $m$ aquí es $F$, esa cosa $F$ también será $G$.

¿La teoría de relocalización da como compromisos sólo aquellos condicionales cuyos antecedentes se refieran a un lugar adonde un sujeto podría viajar, consistentemente con las leyes de la naturaleza, o no hay 
tal restricción? Si no hay tal restricción, tenemos que suponer que alguien que juzga que todos los $F$ son $G$ está comprometido con contrafácticos relativos a lo que pasaría si fuese a un lugar al que pudiese llegar sólo viajando en algún momento anterior más rápido que la luz. Es difícil creer que la pregunta, sumamente oscura, de cómo han de ser evaluados tales contrafácticos tiene que ser decidida antes de que uno pueda creer racionalmente que todos los $F$ son $G$. Así pues, supongamos que el teórico de la relocalización señala mejor, como compromisos, sólo aquellos condicionales cuyos antecedentes mencionan lugares que el creyente podría alcanzar. Entonces el problema es que estos compromisos no determinan las condiciones de verdad. Su satisfacción requeriría que todos los $F$ que estuvieran en lugares que el pensador pudiera alcanzar, fueran $G$; ellos no requerirían que cualesquiera $F$ fuesen $G$. Así, considérese a alguien que cree que todos los planetas que pudiese encontrar, que no sean la Tierra (en un sistema solar u otro), están deshabitados: no necesita creer también que todos los planetas que no sean la Tierra están deshabitados, pero de acuerdo con la última versión de la teoría de relocalización, consideraríamos que lo cree. ${ }^{26}$

26 Como podría anticiparse, esas dificultades también se le plantean a la semśntica de los juegos teóricos para los cuantificadores de Jaakko Hintikka. Esta semántica contiene cláusulas como:

(G.E.) Si [una fórmula] $G$ es de la forma $(\exists x) A$, elijo un miembro de (el dominio) $D$, dándole un nombre, digamos ' $n$ ' (si no lo tenfa antes). El juego continúa con respecto a $A(n / x)$... el resultado de sustituir ' $n$ ' por ' $x$ ' en $A$.

(Logic, Language-Games and Information (Clarendon Press, Oxford, 1973), 100: he cambiado la notación en puntos irrelevantes.) Hintikka añade:

Debe hacerse abstraccion de todas las limitaciones humanas. Debe considerarse al investigador en cuestion, si no como omnipresente, por lo menos como "velocisimo", libre de todas aquellas limitaciones de acceso a las que estamos sometidos todos los humanos (ibid., p. 103). 
La Explicación Revisada trata los objetos que son accesibles al pensador y los que no lo son de modo uniforme con respecto a su relevancia para la cuantificación no-restringida; si se piensa que los lugares inaccesibles tienen ciertas relaciones espaciales con el pensador, aún estarían dentro del rango de sus cuantificadores. ${ }^{27}$

La Explicación Revisada fue delineada para objetos físicos existentes actualmente. Pero es una instancia de una forma general que puede aplicarse a algunos otros rangos de objetos. En el caso de la cuantificación so-

Esta observación la motiva el deseo de que la semántica nos dé las condiciones de verdad clásicamente correctas para las oraciones cuantificadas. Considérese la oración "Todos los $F$ son $G$ ", y supóngase que es falsa, pero únicamente porque algunos $F$ inaccesibles no son $G$ : todos los $F$ accesibles son $G$. Al recorrer las reglas semánticas de Hintikka, vemos que para que "Todos los $F$ son $G$ " sea verdadera, ha de ser verdad que no hay nada que podamos encontrar o elegir que no sea $G$. (Aquí estoy asumiendo la veraión según la cual la naturalesa no se personaliza, y en la cual las matrices universalmente cuantificadas se reescriben como la cuantificación existencial negada de la negación de la matriz dada: véase ibid., p. 101.) Sin la propiedad imaginada de ser "velocísimos", ésta podrla ser verdad, y in embargo, la cuantificación universal "Todos los $F$ son $G^{\prime \prime}$ ser falsa. Ahora bien, puesto que de hecho no somos "velocisimos", como ha insistido Dummett repetida y convincentemente, cualquier explicación de los cuantificadores que mencione esa propiedad tendrá que decir qué características de nuestro pensamiento le dan las mismas condiciones de verdad que las de los pensamientos y expresiones de los seres imaginados que la tienen. (Incluso si Hintikka no aceptara el argumento de Dummett, sigue habiendo una dificultad interna en su semántica. Si fuésemos velocísimos, podríamos viajar más rápido que la luz. Pero la oración "Nada viaja más rápido que la lus" es realmente verdadera: ¿cómo puede la semántica de Hintikka reconocer que es verdadera? Podríamos lograr el mismo resultado con "nadie es velocísimo".)

27 Si adoptamos la Explicación Revisada querremos decir que el sentido en el cual no podemos encontrar un objeto de una clase particular cuando una cuantificación universal es verdadera, donde esto se sustrae de todas las limitaciones humanas, tiene que ser explicado él mismo apelando a la existencia o no existencia de un lugar con un objeto de la clase dada localizado en él -y no la inversa. Queda, por supuesto, como una cuestión para elaboración posterior, la de hasta qué punto tenemos la concepción de lugares y tiempos inaccesibles, tales como aquellos fuera de nuestros conos de luz presentes y de los de nuestros ancestros: pienso discutir esto en otro lugar. 
bre tiempos, por ejemplo, quisiéramos igualmente decir que concebimos este dominio como cerrado bajo operaciones tales como "El tiempo que guarda la relación temporal $T$ con $t^{\prime \prime}$ : si la operación, aplicada a cualquier tiempo que esté ya en el dominio, da algún valor, ese valor también está en el dominio. Nuevamente, el concepto de las relaciones temporales relevantes $T$ es indef inidamente extensible. En algunos otros dominios, por características especiales del rango de objetos en cuestión, no necesitamos apelar a la extensibilidad indefinida en la determinación del rango: sabemos que todos los números naturales, por ejemplo, serán capturados por el ancestral de la función sucesor aplicada a 0 . Así, podemos decir que alguien que juzga "Todos los números naturales son $G^{\text {n }}$ está comprometido con todas las instancias de "Si $n$ es sss...s 0 , entonces $n$ es $G$ ", donde el número de aplicaciones de la función sucesor ' $s$ ' es cualquier número natural; cualesquiera otros compromisos del juicio son derivados de esta familia de compromisos. El uso de la frase "cualquier número natural" en la mitad del enunciado precedente no está dentro del alcance de ninguna actitud atribuida al pensador: la usamos desde fuera, al describir sus compromisos judicativos.

La Explicación Revisada admite un nivel de uniformidad en la descripción de la cuantificación universal sobre objetos concretos y números naturales. En particular, no hace del conocimiento de lo que constituiría una prueba de un contenido de la forma "Todos los números naturales son $G^{n}$ un prerrequisito para la comprensión de tales contenidos. Al adoptar la Explicación Revisada, tomamos por lo que vale la situación de alguien que entiende los cuantificadores sobre números naturales, pero que no sabe cómo se probarían tales contenidos - la situación de todos nosotros antes de que 
descubriésemos 0 aprendiésemos las pruebas inductivas. Lo que aprendemos cuando se enseña este método de prueba es que ciertas premisas garantizan la verdad de un contenido comprendido previamente. ¿Es deficiente el entendimiento de la cuantificación sobre todos los números naturales que posee alguien que no sabe de la prueba inductiva? No querremos insistir generalmente en medios concluyentes para establecer cuantificaciones, ya que esto no lo tendrá la cuantificación a posteriori sobre objetos concretos. Tampoco se requiere el conocimiento de las condiciones de prueba para entender, o para establecer, contenidos complejos en los que están encajadas cuantificaciones universales. Si la condición de verdad está realmente determinada por los compromisos canónicos, un pensador que no sabe nada de las condiciones de prueba en el caso de la aritmética, puede ser capaz de apreciar que si sus compromisos al juzgar

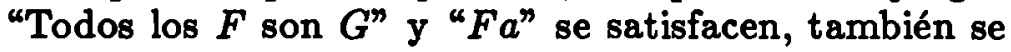
satisfarán los compromisos en los que incurre al juzgar

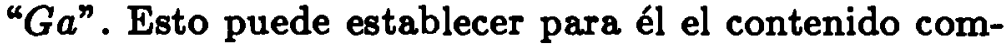
plejo "Si todos los $F$ son $G$ y $a$ es $F$, entonces $a$ es $G$ ", sin saber ninguna de las condiciones de prueba para la cuantificación universal.

La Explicación Revisada no tiene una extensión natural para la cuantificación universal sobre los números reales, o sobre totalidades grandes de conjuntos. Puede haber, entonces, una explicación más general de la cuantificación universal que sea aplicable a todos los dominios, sean cuales sean. La Explicación Revisada es, en el mejor de los casos, parcial. Pero aún podemos esperar que, para las áreas de las que se ocupa, la Explicación Revisada sea una consecuencia de la explicación más general cuando se le toma junto con las características especiales de estas áreas.

Asumiré la Explicación Revisada como mi elabora- 
ción oficial, aunque parcial, de una explicación neo-ramseyana de los contenidos de la cuantificación universal. Esto se ajusta a una de las formas generales que mencioné antes, $y$ a la que pretendí adherirme, a saber, que los compromisos canónicos determinan la condición de verdad clásica, en el sentido de que aquellas condiciones para un pensamiento dado se satisfacen todas si y sólo si el pensamiento es verdadero. Al dar la explicación neo-ramseyana, no he hecho uso de la idea de un humano, o algún pensador ideal, que sea capaz de reconocer que se da la condición de verdad para un cuantificador universal. Dummett ha escrito:

ya que... de la suposición de que se da la condición para la verdad de un enunciado matemático, entendido platónicamente, no puede en general inferirse que es tal que necesita suponerse que un ser humano es capas de reconocer que se da, no podemos darle peso a la concepción de que tenemos un conocimiento implícito de cuál es esa condición, ya que nada que hagamos puede equipararse a una manifestación de tal conocimiento. ${ }^{28}$

La Explicación neo-ramseyana sugiere que la condición clásica de verdad está manifestada no por las acciones de un pensador cuando reconoce que la condición de verdad se da, sino más bien por sus compromisos (manifestables) cuando juzga la cuantificación universal: para que esos compromisos se satisfagan, la condición clásica de verdad tiene que darse, aunque no es conocimiento conclusivo que así lo haga. Dummett considera las condiciones clásicas de verdad para las cuantificaciones universales como proyectadas ilegítimamente

28 Elements of Intuitionism (Clarendon Press, Oxford, 1977), p. 375. Véase también au Frege: Philosophy of Language (Duckworth, Londres, 1973), 465: "en una concepción realista del significado, la comprensión de una oración consiste en el conocimiento de aquello que tiene que ser el caso para que ella sea verdadera; y tal conocimiento debe, a su ves, consistir en un modelo de lo que serfa reconocer la oración como verdadera por los medios más directos". Es el "debe" el que estoy cuestionando aquí. 
a partir de casos observacionales, donde sostiene que es legítimo y correcto hablar de la habilidad de un pensador para reconocer que se da la condición de verdad para un enunciado. ${ }^{29}$ Pero de acuerdo con la explicación presente, el caso observacional mismo despliega la misma estructura que la explicación neo-ramseyana de la cuantificación. El pensamiento "Esa caja es cúbica" tiene la condición de verdad que tiene porque los compromisos contrafácticos constitutivos del pensador - grosso modo, que en circunstancias adecuadas la caja sería percibida como cúbica desde diferentes ángulos- se satisfacen si y sólo si la caja realmente sí tiene la forma de un cubo. Cuando reflexionamos sobre el caso observacional, parece que no hay necesidad de apelar al reconocimiento, por parte de un pensador, de que una condición de verdad se da para que haya manifestaciones de la comprensión de una condición de verdad clásica: y tampoco una posibilidad de apelar así cuando reflexionamos sobre los cuadriláteros irregulares, etc. En la explicación neo-ramseyana, la cuantificación universal es sólo otra ilustración de este punto general. Hay características especiales peculiares respectivamente a conceptos observacionales y a cuantificadores; y hay maneras equivocadas de defender las condiciones de verdad clásicas, muchas de las cuales han sido criticadas por Dummett; pero aún queda en la presente teoría del contenido un nivel en que, respecto de las relaciones entre verdad y evidencia, los contenidos observacionales y de cuantificación universal son estructuralmente idénticos.

3. La Conjetura general que formulamos al principio de este trabajo puede hacerse más convincente sólo mediante la investigación detallada de muchos otros tipos

"What is a Theory of Meaning? (II)"; especialmente pp. 98 y ss. 
problemáticos de contenido. El modelo de un contenido $p$ y sus fundamentos canónicos asociados $G(p)$ o compromisos canónicos $C(p)$ no es de ninguna manera sacrosanto para llevar a cabo este programa. Es plausible que se necesite algo diferente donde el concepto en cuestión esté gobernado por principios inferenciales. También puede ser que para algunos contenidos -quizá contenidos acerca de lugares y tiempos inaccesiblesnecesitemos basarnos en condiciones de verdad de contenidos para los cuales ya tenemos una implementación plausible del programa: esos serían, entonces, casos a los que se aplicaría la segunda cláusula de nuestra conjetura inicial. Pero todo esto es especulativo y tentativo. Respecto del programa general, concluyo con dos observaciones: Primero, los casos que hemos considerado sugieren que no puede haber un argumento general razonable para mostrar que las condiciones de aceptación no logran determinar condiciones de verdad realistas. Segundo, no he dudado que la atribución correcta de una condición de verdad particular debe manifestarse en el pensamiento y la acción de aquel al que se le atribuye. La posición hacia la que me he estado moviendo puede resumirse así: manifestacionismo sin verificacionismo.

Traducción de Silvia Bello y Enrique Villanueva 


\section{SUMMARY}

In this paper a question is asked about what the relation is between the acceptance conditions of what is believed and the conditions for what is believed to determine the truth value true. By an "acceptance condition" of a given content, it is meant a correct statement of a normative condition concerning that content, and it is argued in support of the conjeture that there is a conception of a content's acceptance conditions on which those acceptance conditions determine its truth conditions. The position towards which the arguments run is summarized as "manifestationism without verificationism".

It is argued for the conjeture first in the case of certain observational contents and next in connection with universally quantified contents. 